\title{
60-W 946-nm cryogenically-cooled Nd:YAG laser
}

\author{
S. Cante ${ }^{1}$ (D) S. Valle ${ }^{1} \cdot$ S. J. Yoon ${ }^{1} \cdot$ J. I. Mackenzie ${ }^{1}$
}

Received: 10 April 2019 / Accepted: 17 June 2019 / Published online: 27 June 2019

(c) The Author(s) 2019

\begin{abstract}
Detailed spectroscopic characterisation of the ${ }^{4} I_{9 / 2} \rightarrow{ }^{4} F_{3 / 2}$ absorption cross section of Nd:YAG at temperatures ranging from room temperature to liquid nitrogen temperature is reported. Exploiting this in-band pumping scheme, a novel volumeBragg-grating-locked diode-laser array (DLA) operating at $869 \mathrm{~nm}$ was developed to end-pump a cryogenically cooled $\mathrm{Nd}$ :YAG crystal. As a result, the first 60-W cryogenically-cooled Nd:YAG laser operating at $946 \mathrm{~nm}$ is demonstrated with a slope, and optical-to-optical, efficiency of $52 \%$.
\end{abstract}

\section{Introduction}

Fundamentally limited by low gain $[1,2]$ and detrimental thermo-optical effects at room temperature (RT), which are exacerbated by additional heating from energy transfer upconversion (ETU) and cross relaxation (CR) [3], the performance improvements in recent years for the quasifour-level (Q4L) 946-nm Nd:YAG laser have saturated. The output power of this laser system is primarily constrained by the limits of pump brightness and dynamic thermally induced losses associated with the crystal and its geometry. To date, the best demonstrations of laser performance for this transition in a bulk configuration report $16 \mathrm{~W}$ of output power for a rod [4], $34 \mathrm{~W}$ for a fibre-rod [5], and $27 \mathrm{~W}$ for a fibre-laser [6]. In the thin disk and planar waveguide laser architectures instead, $20 \mathrm{~W}$ [7] and $105 \mathrm{~W}$ [8] have been achieved, respectively. These demonstrations stand as the state-of-the-art for the Q4L sub-1 $\mu \mathrm{m}$ transition of the $\mathrm{Nd}^{3+}$ impurity, which as shown in the respective references, is dominantly in the YAG host.

Exploited since the earliest laser demonstrations [9], cryogenic cooling of the gain medium has proved to be an effective way of power- and energy-scaling solid-state-lasers [10], mainly due to an enhancement of spectroscopic and

This article is part of the topical collection "Cryogenically Cooled Lasers" guest edited by Jacob I Mackenzie, Nikolay TerGabrielyan and Yung Fu Chen.

S. Cante

s.cante@soton.ac.uk

1 Optoelectronics Research Centre, University of Southampton, Southampton SO17 1BJ, UK thermo-optical properties [11, 12]. The most commonly used dopant in this cryogenically-cooled regime is $\mathrm{Yb}^{3+}$, which, thanks to a low quantum defect $(\sim 9 \%)$ coupled with a reduction in reabsorption losses and an overall improvement in spectroscpic properties, has been reported at the multi-hundreds-of-Watts in CW bulk systems $[12,13]$, and up to $2.6 \mathrm{~kW}$ of peak power for a master-oscillator poweramplifier (MOPA) system [14].

Potential materials-processing applications at wavelengths shorter than $1 \mu \mathrm{m}$, which can be frequency up-converted to wavelengths in the blue-green and DUV spectral regimes, motivate our study for power- and energy-scaling of the ${ }^{4} I_{9 / 2} \rightarrow{ }^{4} F_{3 / 2}$ laser transition of Nd:YAG. Furthermore, considering only the ground and first excited states, akin to the $Y b^{3+}$ energy scheme, this Q4L system if pumped inband, for example at $869 \mathrm{~nm}$, offers a QD 8\%, three times smaller than when pumping at $808 \mathrm{~nm}$, traditionally used as the strongest absorption feature. Therefore, the potential performance enhancement for the aggressively cooled 946-nm Nd:YAG laser promises to be comparable with that obtained with Yb:YAG lasers, as the first demonstrations suggest [15, 16], since the spectroscopic and physical parameters that limit the performance at RT are also dramatically improved by cryogenic cooling. Notwithstanding, there are significant additional technical challenges to address, such as strong gain competition with the dominant ${ }^{4} F_{3 / 2} \rightarrow{ }^{4} I_{11 / 2} 1 \mu \mathrm{m}$ transition and a restriction in the $\mathrm{Nd}^{3+}$ doping concentration due to enhanced non-radiative decay channels and heating of the host. It is important to characterise such nonlinear processes, such as ETU and CR, for completeness in performance models [3, 17-19]. 
Driven by the above-mentioned motivations, in this work, we undertake a comprehensive investigation of ground-state absorption to the ${ }^{4} F_{3 / 2}$ energy level, for temperatures spanning from RT to LNT. We determined that the absorption peak around $869 \mathrm{~nm}$ is the strongest of the R-band, according to the nomenclature of [20], and becomes of comparable magnitude to the RT $808 \mathrm{~nm}$ absorption peak.

This characterisation was the impetus for the development of a novel volume Bragg grating (VBG)-locked diode-laserbar source operating at $869 \mathrm{~nm}$, with a spectral bandwidth of $(0.20 \pm 0.02) \mathrm{nm}$, matching the sharp absorption peak at LNT. We employ this source to pump a cryogenically-cooled $\mathrm{Nd}$ :YAG laser crystal, leading to a maximum 946-nm output power of $60 \mathrm{~W}$, with a $52 \%$ slope, and optical-to-optical, efficiency. Further power-scaling potential is discussed for this equivalent energy scheme to the $\mathrm{Yb}^{3+}$ rare earth ion.

\section{Experimental setup}

\subsection{Absorption cross section measurement}

Due to its importance for in-band or thermally-boosted pumping [21], the absorption cross section of Nd:YAG corresponding to ground-state absorption (GSA) transitions from the ${ }^{4} I_{9 / 2}$ energy level $\left(Z_{1-5}\right)$ to the metastable ${ }^{4} F_{3 / 2}$ first excited state energy level $\left(\mathrm{R}_{1,2}\right)$, has been well characterised at RT. Here, we present a thorough characterisation of the GSA over the range of temperatures from RT to LNT via simple small-signal absorption measurements.

The setup employed is depicted in Fig. 1. In order to cover the wavelengths of interest, a high-power LED (Roithner, Lasertechnik JET-870-05) was selected, emitting in the range 820-900 nm. Although having significantly more power spectral density than a white-light source, the relatively low brightness of the LED meant it had to be operated in a QCW regime, with $10 \mu$ s pulses and a $10 \%$ duty cycle, to reach $150 \mathrm{~mW}$ of total peak power. Notwithstanding, when coupled to an $200 \mu \mathrm{m}, 0.22$ NA optical fibre, only $1 \mathrm{~mW}$ could be extracted from the exit facet. Using a $3 \times$ afocal beam-expander, a spot size of $600 \mu \mathrm{m}$ diameter was generated in a $3 \mathrm{~mm}$ long, 1 at.\%-doped, Nd:YAG sample. The transmitted light was then coupled into an Optical Spectrum

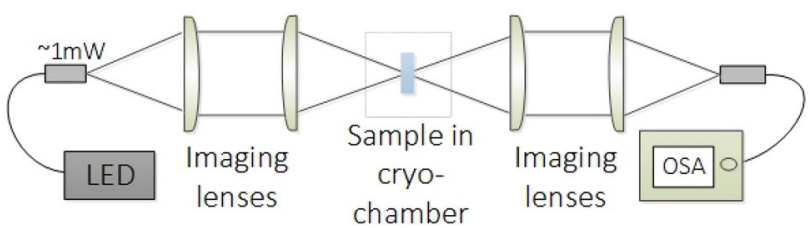

Fig. 1 Absorption cross section measurement setup
Analyser (OSA, ANDO-AQ6317B) through a second reimaging telescope and $300 \mu \mathrm{m}, 0.22 \mathrm{NA}$, patch fibre.

During the experiment, the Nd:YAG sample was wrapped into indium foil, with a spring loaded clamp keeping the crystal in thermal contact with the cryo-cooled cold head, as in [22].

The probe's spectrum was measured with a resolution of $0.1 \mathrm{~nm}$, and a sampling step of $0.01 \mathrm{~nm}$, with and without the sample in place, and the absorption cross section spectrum calculated using Beer's law, as in [19]. Losses due to coating reflections and scattering were accounted for by determining the transmission of out of band wavelengths. Absorption measurements were then executed for several different temperatures between RT and LNT.

\subsection{Diode pump setup}

From the absorption measurements it was found that the $869 \mathrm{~nm}$ transition was sufficiently strong at cryogenic temperatures to make it an effective pumping wavelength.

A customised pump was developed based upon an actively cooled 200-W DLA from DILAS, with a nominal wavelength of $873 \mathrm{~nm}$. The output of the DLA was initially conditioned using a Beam Transformation System (BTS) [23]. This micro-optic provided fast-axis collimation, coupled with rotation of the beams from each individual emitter in the DLA by $90^{\circ}$, namely "fast" $\rightarrow$ "slow" axes (as defined by the diode-laser). Here, we define the $\mathrm{x}$-coordinate as the slow-axis, and the y-coordinate as the fast-axis with reference to the DLA, respectively. While the $\mathrm{z}$-axis is parallel to the direction of laser propagation. After the BTS, the beam divergence in the y-axis is nominally that of the individual emitters' slow axis, for which the collection of beamlets can be collimated with a single cylindrical lens. However, to enhance the locking efficiency a VBG was placed immediately after the BTS. Following which, cylindrical lenses were employed to manipulate and collimate the beam in the $\mathrm{x}$ - and y- axes separately, as shown in Fig. 2.

A cylindrical lens, $\mathrm{F}_{y}=40 \mathrm{~mm}$, collimated the DLA output in the $\mathrm{y}$-axis; instead the $\mathrm{x}$-axis was collimated by the BTS, and lenses $F_{x_{1}}=75 \mathrm{~mm}$ and $F_{x_{2}}=130 \mathrm{~mm}$ provided a $1.7 \times$ imaging telescope. A typical polarisation combining setup was employed to chop the $\mathrm{x}$-axis beam in half;

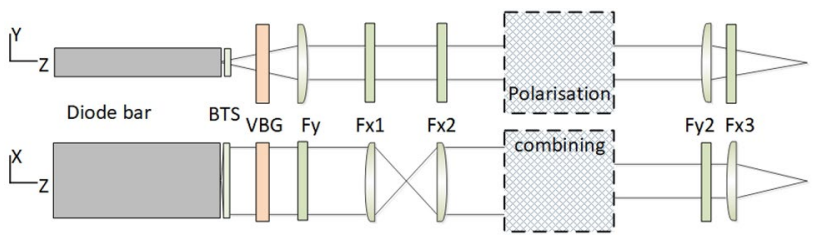

Fig. 2 Layout of the optical components for $\mathrm{x}$ - and $\mathrm{y}$-axes 
consequently, the beam quality for this axis was approximately halved as well.

\subsection{Laser cavity}

A $3 \times 3 \times 15 \mathrm{~mm}^{3}, 0.3$ at $\% \mathrm{Nd}: Y A G$ crystal was used for the laser experiment. The smaller facets were anti-reflection coated for the pump and laser wavelengths. Wrapped in $200-\mu \mathrm{m}$-thick indium foil, the crystal was clamped between two copper plates. One plate was bolted directly to the coldhead of a liquid-nitrogen dewar, the assembly providing cooling through two side faces of the crystal. A copper braid brazed to both pieces was employed to ensure a thermal link between the two copper parts. The assembly was isolated from the surrounding environment via $\mathrm{a} \sim 10^{-7} \mathrm{mbar}$ vacuum, and the vacuum chamber was equipped with $10 \mathrm{~mm}$ thick windows that allowed optical access to the crystal while acting as sealing plates; these windows were ARcoated and had a $98.4 \%$ and $99.9 \%$ nominal transmission at the pump and laser wavelengths. The temperature of the system was monitored by two temperature sensors positioned on the side of the cold head and on the $\mathrm{Cu}$ piece further from the cold head, respectively.

We employed a simple plano-concave $168.5 \mathrm{~mm}$ long linear cavity, as shown in Fig. 3, comprising a flat input mirror, and a $200 \mathrm{~mm}$ radius of curvature (ROC), $T=10 \%$ output coupler. The input mirror was positioned in close proximity to one window, resulting in a distance from mirror to crystal being $52.5 \mathrm{~mm}$. This configuration provided a fundamental laser mode of $\sim 190 \mu \mathrm{m}$ over a thermal lens focal length range from infinity to $\sim 500 \mathrm{~mm}$.

\section{Results and discussion}

\subsection{Absorption cross section results}

The strongest absorption peak of the R-band (860-900 nm) is at $869 \mathrm{~nm}$ (see Fig. 4), representing the transition from the $\mathrm{Z}_{0}$ to $\mathrm{R}_{2}$ Stark levels.

The strongest $\mathrm{R} \rightarrow \mathrm{Z}$ absorption cross section peak, centred at $(868.95 \pm 0.02) \mathrm{nm}$ at $\mathrm{RT}$, increases from $(2.32 \pm 0.11) \mathrm{pm}^{2}$ to $(6.54 \pm 0.33) \mathrm{pm}^{2}$, narrows from $(0.87 \pm 0.05) \mathrm{nm}$ to $(0.27 \pm 0.05) \mathrm{nm}$, and blueshifts to

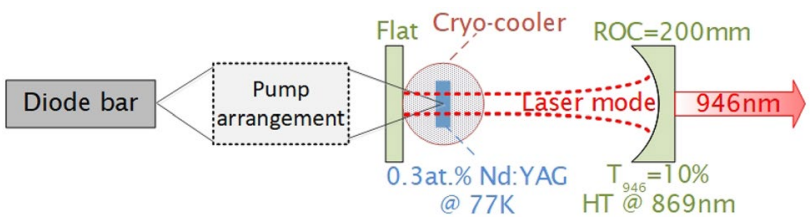

Fig. 3 Linear plano-concave cavity

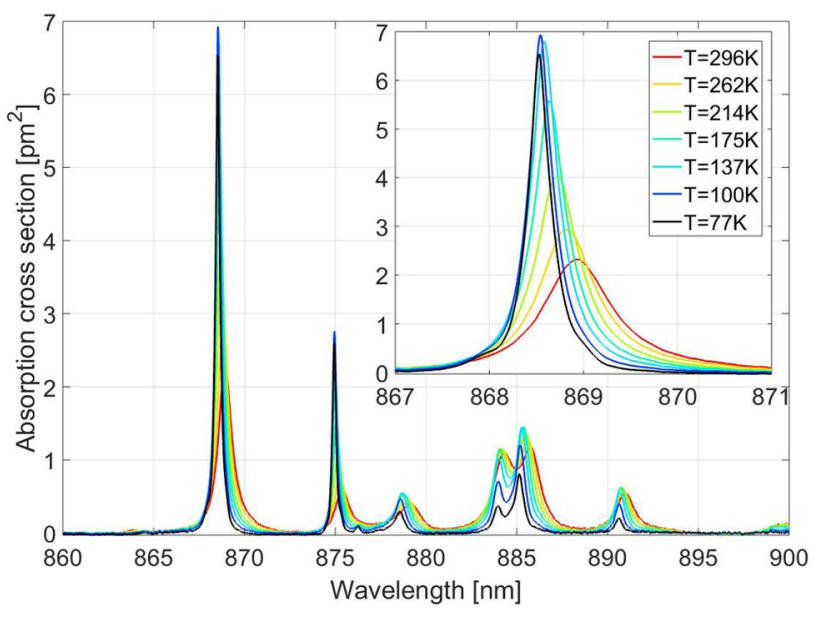

Fig. 4 Temperature-dependent calculated absorption cross section from measurement of probe transmission for transitions between Rand Z-bands for Nd:YAG. Inset: zoom on the peak at $869 \mathrm{~nm}$ (axes' units are the same as the main plot's)

$(868.53 \pm 0.02) \mathrm{nm}$ over the temperature range from RT to LNT, as shown in Fig. 5.

\subsection{Pump characterisation}

A key driver in the performance of 946-nm Nd:YAG lasers is pump brightness. We characterised the DLA beam quality at $P_{\text {out }}=100 \mathrm{~W}$ after the BTS, which was found to be $M_{x}^{2}=62 \pm 1$ and $M_{y}^{2}=20 \pm 1$ in the $\mathrm{x}$ - and $\mathrm{y}$-axes, respectively. The spot size and the relative beam quality in the $y$-axis increased substantially with drive current, as the higher-order modes of each of the broad-stripe emitters only filled out to the full width of the waveguide for pump powers $>50 \mathrm{~W}$. In contrast, the orthogonal fast-axis collimated beam properties in the $\mathrm{x}$-axis remained relatively constant. A VBG, with $20 \%$ reflectivity at $868.2 \mathrm{~nm}$ and $0.12 \mathrm{~nm}$

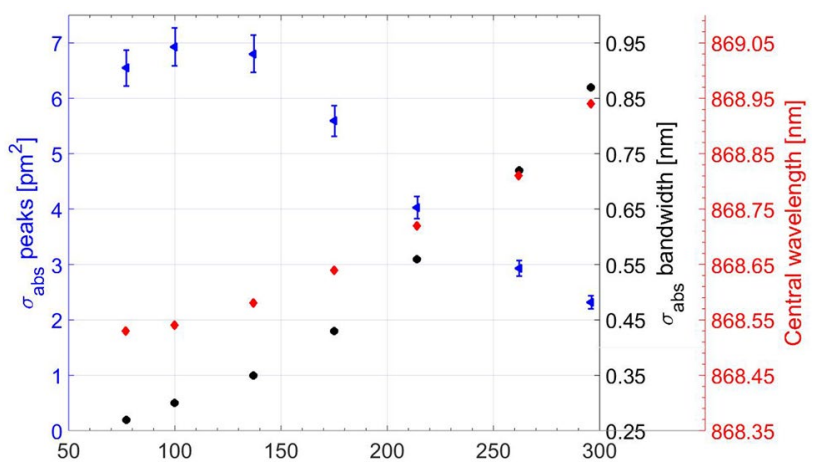

Fig. 5 Left axis (blue triangles): absorption cross section peak values versus temperature; right axis (black circles): bandwidth of the respective peaks versus temperature; right axis (red diamonds): central wavelength of the respective peaks versus temperature 
FWHM bandwidth, was mounted in an adjustable mount, positioned at $<2 \mathrm{~mm}$ from the BTS; its orientation was optimised in order to provide optimal locking of the DLA wavelength, monitored via the OSA. The bandwidth of the source changed from $(2.4 \pm 0.1) \mathrm{nm}$ without the VBG-locking to $(0.22 \pm 0.01) \mathrm{nm}$ with, whereas the beam qualities for the respective axes became $\mathrm{M}_{x}^{2}=63 \pm 1$ and $\mathrm{M}_{y}^{2}=22 \pm 1$.

As the photo-thermo-refractive glass has a finite absorption at the wavelength of interest, increasing diode-laser power and linear expansion of the VBG induced a temperature-dependent chirp of the central VBG-locked wavelength equal to $3.3 \mathrm{pm} / \mathrm{W}$, as illustrated in Fig. 6. The locking wavelength at RT was designed so that the overlap between the locked wavelength and the absorption peak around $869 \mathrm{~nm}$ at LNT would be optimal at the maximum output power of the DLA.

The absorption efficiency was measured by monitoring the transmitted pump power through the laser crystal and cavity output coupler (highly transmissive, HT, at the pump wavelength), while a second dichroic mirror (highly reflective, HR, at $946 \mathrm{~nm}$ and HT at $869 \mathrm{~nm}$ ) was used to reject the $946-\mathrm{nm}$ radiation.

When VBG-locked, the pump absorption efficiency in the Nd:YAG crystal increased almost linearly from 20 to $95 \%$ for incident powers, measured before the cryostat's window, from 3.7 to $58 \mathrm{~W}$ with a rate of $1.15 \% / \mathrm{W}$, associated with the pump-wavelength chirp. The absorbed power clamped at $99 \%$ for incident powers from 60 to $120 \mathrm{~W}$ during lasing (see Fig. 7). This demonstrated the need to ensure that the small chirp in the VBG wavelength with increasing power is accounted for.

The beam quality of the output beam was determined using a CCD camera (Spiricon BGP-USB-SP503U) positioned on a computer controlled translation stage, measuring

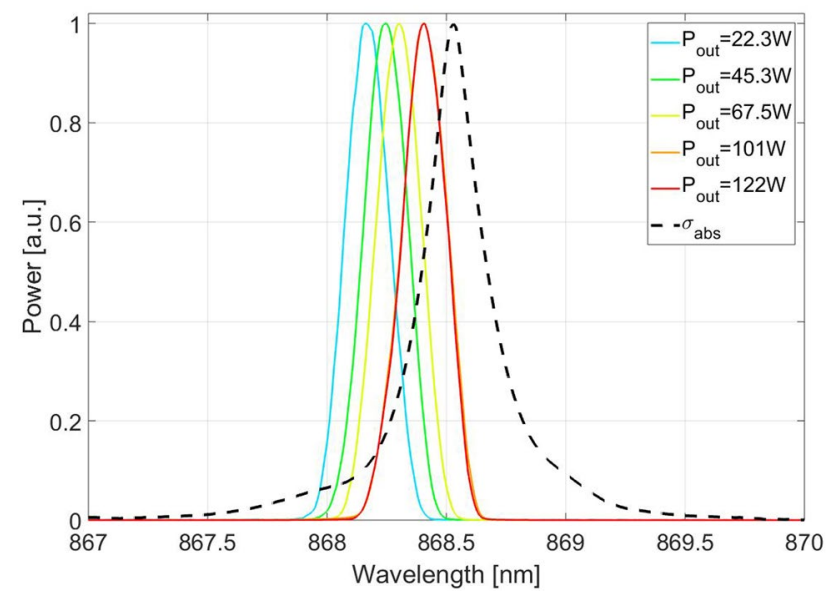

Fig. 6 Central VBG-locked DLA's wavelength chirp for different output powers. Dashed black line: Nd:YAG's $869 \mathrm{~nm}$ normalised absorption cross section peak at LNT

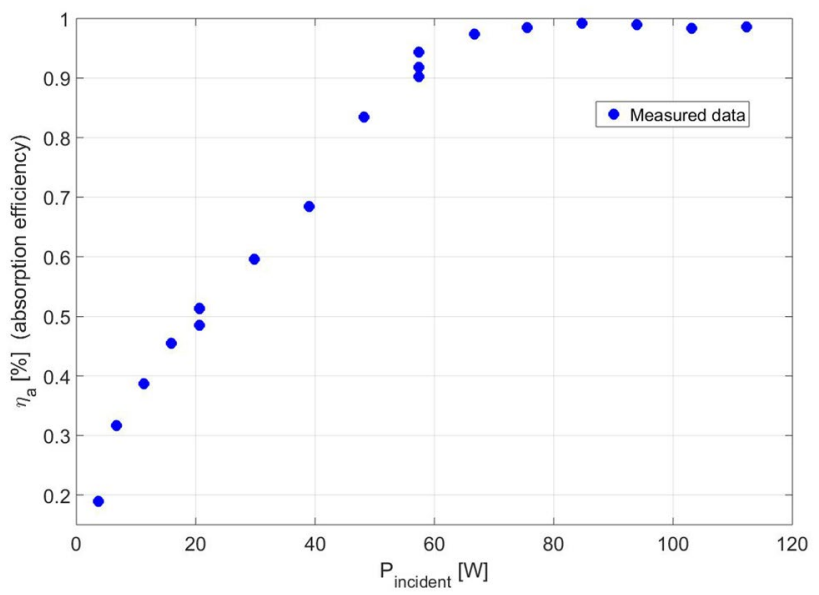

Fig. 7 Absorption efficiency of the VBG-locked pump for incident powers from 4 to $120 \mathrm{~W}$ during lasing

the second moments $D_{4 \sigma}$ beam caustic of the attenuated laser created by a $f=250 \mathrm{~mm}$ focusing lens. Camera setup, data acquisition, and statistical analysis were performed in conjunction with the stage control to ensure optimal measurement of the beam parameters and clean caustic measurements.

The measured beam qualities for the respective axes after the polarisation combining setup were $\mathrm{M}_{x}^{2}=33 \pm 1$ and $\mathrm{M}_{y}^{2}=21 \pm 1$. For the focusing of the pump light into the laser crystal we used two separate cylindrical lenses of $200 \mathrm{~mm}$ focal length for the respective $\mathrm{x}$ - and $\mathrm{y}$-axes, $\left(\mathrm{F}_{y_{2}}\right.$, $\mathrm{F}_{x_{3}}$ ) to produce a anastigmatic beam waist. The final beam qualities and spot sizes at $120 \mathrm{~W}$ were $\mathrm{M}_{x}^{2}=33 \pm 1$ and $\mathbf{M}_{y}^{2}=22 \pm 1$, and $w_{0_{x}}=373 \mu \mathrm{m}$ and $w_{0 y}=352 \mu \mathrm{m}$, respectively for the $\mathrm{x}$ - and $\mathrm{y}$-axes as shown in Fig. 8.

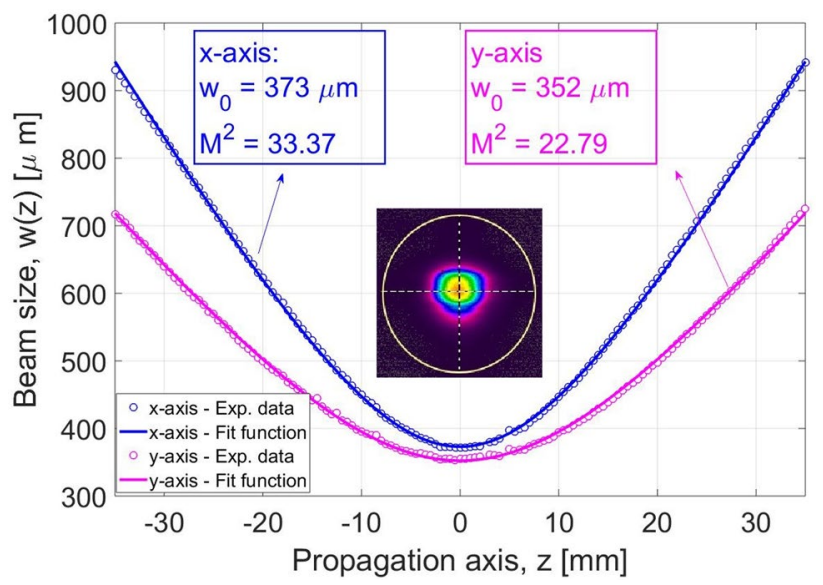

Fig. 8 Beam quality and spot sizes in air after the focussing lenses 


\subsection{6-nm cryogenically-cooled laser results}

The temperature of the cold head was fixed at $77 \mathrm{~K}$ by the LN bath, and monitored throughout the experiment by the two temperature sensors. A good indicator of the average temperature of the crystal was the temperature-dependent emission wavelength [22], which was measured in real time by the OSA after capturing the light scattered off the thermal-detector used to measure the output power.

The laser threshold was measured to be $2 \mathrm{~W}$ of absorbed pump power; at this stage the emission wavelength was $(946.224 \pm 0.005) \mathrm{nm}$, in line with the value at $77 \mathrm{~K}$ given in [22].

The slope, and optical-to-optical, efficiency versus absorbed pump power was measured to be $52 \%$, with a maximum laser output power of $60 \mathrm{~W}$ (Fig. 9).

Employing the same beam profiling methodology described above, we measured the beam quality for the respective axes to be $\mathrm{M}_{x}^{2}=1.60$ and $\mathrm{M}_{y}^{2}=1.08$, at an output power of $1 \mathrm{~W}$. The significant difference between the two axes was due to the pump's beam quality being worse in the x-axis compared with the y-axis, leading to an overlap factor of nearly two, $a_{x}=w_{P_{x}} / w_{L_{x}}$ [2], near threshold. Furthermore, the near threshold overlap in the y-axis, $a_{y} \sim 1.3$, increased to $\sim 1.8$ at pump powers $>50 \mathrm{~W}$. In contrast, the overlap in the $\mathrm{x}$-axis stayed relatively constant throughout the range of available pump output powers.

The beam quality for the respective axes degraded with increasing laser power: for $P_{\text {out }}=40 \mathrm{~W}$, we measured $\mathrm{M}_{x}^{2}=3.10$ and $\mathrm{M}_{y}^{2}=2.32$, with modal instability affecting the precision of the measurement, as can be appreciated in Fig. 10. For output powers higher than $40 \mathrm{~W}$ the measured beam size was fluctuating significantly, and as such a stable $\mathrm{M}^{2}$ measurement could not be achieved.

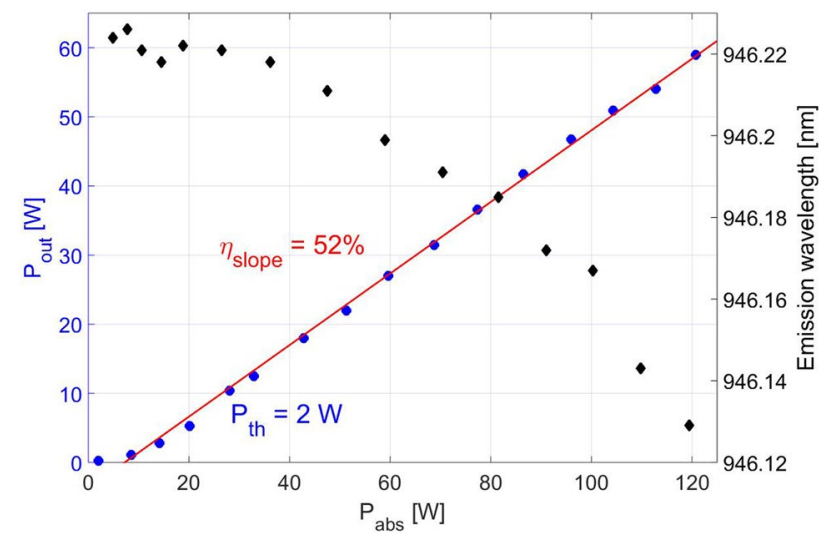

Fig. 9 Right axis (black diamonds): laser emission wavelength versus absorbed pump power. Left axis (blue dots): slope efficiency versus absorbed pump power for the 60-W, 946-nm, cryogenically-cooled $\mathrm{Nd}$ :YAG laser developed
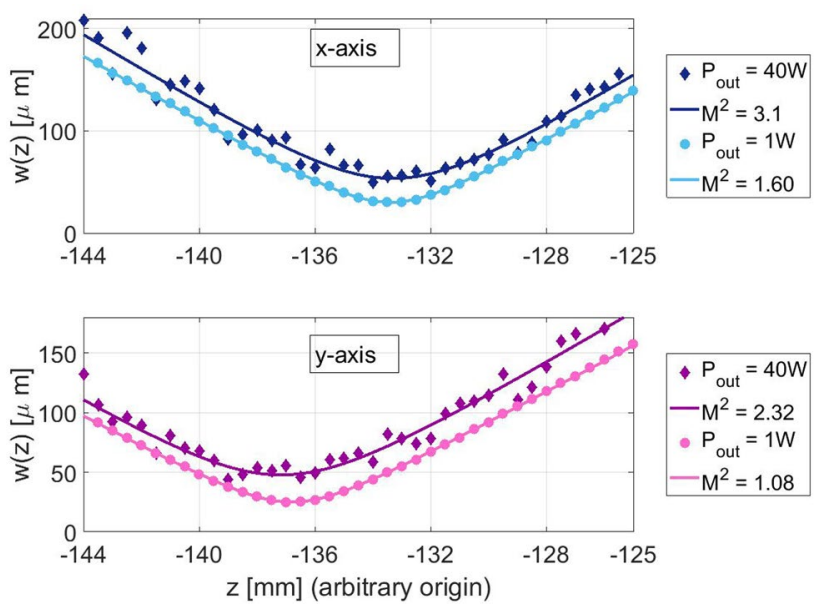

Fig. 10 Measured beam qualities for the respective axes for $P_{\text {out }}=1$ $\mathrm{W}$ (circles) and $P_{\text {out }}=40 \mathrm{~W}$ (diamonds)

Simultaneous to the beam quality degradation, we recorded a strong blueshift in the emission wavelength, reaching $(946.129 \pm 0.005) \mathrm{nm}$ when $P_{\text {out }}=60 \mathrm{~W}$. From this we estimate that the average local temperature rise, i.e. in the cavity-mode volume within the crystal, was $\sim 80 \mathrm{~K}$. This value is substantially higher than the $\sim 20 \mathrm{~K}$ predicted via an analytical model, which includes the temperaturedependent thermal conductivity of the crystal [24]. The fact that the temperature sensors only measured an increase of a few degrees in the $\mathrm{Cu}$ mount and cold-head of the cryostat, suggested the existence of a large temperature gradient across the crystal-mount interface. In fact, as discussed in the aforementioned model [24], due to the temperature dependence of the crystal's thermal conductivity, a significant thermal barrier at the boundary causes a temperature rise across it and throughout the crystal. This therefore leads to an additional increase in temperature at the centre of the crystal. Correspondingly, we estimated that the heat transfer coefficient, $h$, between crystal and coolant that would be associated with such a temperature rise is between $(0.25-0.5) \mathrm{W} / \mathrm{K} \mathrm{cm}^{2}$. That is only $4-10$ times lower than the best value reported for RT heat-sinking with a thermal grease between the crystal and a copper mount [25]. It is evident that due to the large gradient in the thermal conductivity dependence on temperature for YAG in the cryogenic regime [24], the peak temperature-rise sensitivity to the magnitude of $h$ is enhanced with comparison to operation at RT. An important consideration for operation of cryogenically cooled solid-state lasers. 


\section{Conclusions}

We have undertaken a thorough investigation of the temperature-dependent R-band absorption cross section in Nd:YAG, which motivated the development of a novel VBG-locked diode-laser pump source at $869 \mathrm{~nm}$. Employing this source, a $99 \%$ absorption efficiency at the maximum pump powers tested was achieved, and the first $60-\mathrm{W}$, cryogenically-cooled 946-nm Nd:YAG laser demonstrated. A slope, and opticalto-optical, efficiency of $52 \%$ was observed, limited by the temperature rise in the pumped volume crystal and a nonoptimal overlap between pump and cavity modes. Further optimisation was not undertaken at this stage, due to the relatively poor heat extraction of the current setup.

An estimated average local temperature rise in the crystal of $\sim 80 \mathrm{~K}$ was determined by monitoring the emission wavelength, suggesting a heat transfer coefficient in the range of $(0.25-0.5) \mathrm{W} / \mathrm{K} \mathrm{cm}^{2}$ between the crystal and the LN coolant. This, coupled to thermally induced optical aberrations, caused modal instabilities that resulted in degradation in beam quality for high output power and eventual termination of laser action.

With improved mounting arrangements, we expect performance can be significantly improved towards the theoretical slope efficiency in excess of $80 \%$ and power levels in the 100's W regime.

Acknowledgements S. Cante and J. I. Mackenzie acknowledge support from EPSRC. Data presented can be found at https://doi.org/10.5258/ SOTON/D0875.

Funding Engineering and Physical Sciences Research Council (EPSRC), Grant EP/M020932/1.

Open Access This article is distributed under the terms of the Creative Commons Attribution 4.0 International License (http://creativeco mmons.org/licenses/by/4.0/), which permits unrestricted use, distribution, and reproduction in any medium, provided you give appropriate credit to the original author(s) and the source, provide a link to the Creative Commons license, and indicate if changes were made.

\section{References}

1. T.Y. Fan, R.L. Byer, IEEE J. Quantum Electron. 23, 605-612 (1987)
2. W.P. Risk, J. Opt. Soc. Am. B 5, 1412-1423 (1988)

3. S. Bjurshagen, R. Koch, Appl. Opt. 43, 4753-4767 (2004)

4. R. Zhou, E. Li, H. Li, P. Wang, J. Yao, Opt. Lett. 31, 1869-1871 (2006)

5. X. Delen, I. Martial, J. Didierjean, N. Aubry, D. Sangla, F. Balembois, P. Georges, Appl. Phys. B 104, 1-4 (2011)

6. P.H. Pax, V.V. Khitrov, D.R. Drachenberg, G.S. Allen, B. Ward, M. Dubinskii, M.J. Messerly, J.W. Dawson, Appl. Phys. B 24, 28633-28647 (2016)

7. J. Gao, J. Speiser, A. Giesen, in Advanced Solid-State Photonics, Optical Society of America, TuB34 (2005)

8. S.P. Ng, J.I. Mackenzie, Appl. Opt. 22, 494-498 (2012)

9. P.P. Sorokin, M.J. Stevenson, Phys. Rev. Lett. 5, 557-559 (1960)

10. D.C. Brown, IEEE J. Quantum Electron. 3, 861-873 (1997)

11. D.C. Brown, IEEE J. Sel. Top. Quantum Electron. 11, 587-599 (2005)

12. T.Y. Fan, IEEE J. Sel. Top. Quantum Electron. 13, 448-459 (2007)

13. D.C. Brown, J.M. Singley, K. Kowalewski, J. Guelzow, V. Vitali, Opt. Express 18, 24770-24792 (2010)

14. T. Morioka, K. Hamamoto, S. Nishikata, N. Inoue, Y. Ohtani, H. Ikebuchi, H. Daigo, Proceedings, SPIE LASE Conference on Solid State Lasers XXVIII (2019)

15. S.J. Yoon, J.I. Mackenzie, Opt. Express 22, 8069-8075 (2014)

16. C.Y. Cho, T.L. Huang, H.P. Cheng, K.F. Huang, Y.F. Chen, Opt. Express 24, 1-8 (2016)

17. S. Cante, S. Valle, J.I. Mackenzie, 8th EPS-QEOD Europhoton Conference (2018)

18. S.J. Yoon, R.P. Yan, S.J. Beecher, J.I. Mackenzie, Opt. Express 5, 926-931 (2015)

19. S. Cante, S.J. Beecher, J.I. Mackenzie, Opt. Express 26, 64786489 (2018)

20. E.H. Carlson, G.H. Dieke, J. Chem. Phys. 34, 1602-1609 (1961)

21. R. Lavi, S. Jackel, Opt. Express 39(18), 3093-3098 (2000)

22. S.J. Yoon, Dissertation (University of Southampton, ORC, 2016)

23. R. Goering, P. Schreiber, T. Possner, Proc. SPIE 3008, Miniaturized Systems with Micro-Optics and Micromechanics II (1997)

24. L. Cini, J.I. Mackenzie, Appl. Phys. B 123, 273 (2017)

25. S. Chenais, F. Druon, S. Forget, F. Balembois, Prog. Quantum Electron. 30, 89-153 (2006)

Publisher's Note Springer Nature remains neutral with regard to jurisdictional claims in published maps and institutional affiliations. 